\title{
Bonding of third additive with Se \& Te alters DC conductivity of ternary ChGs
}

\begin{abstract}
Presented paper investigates the effect of $\mathrm{Pb}$ and $\mathrm{Cd}$ as third additive in $\mathrm{Se}-\mathrm{Te}$ based ternary chalcogenide glasses (ChGs). These glassy samples, prepared by rapid cooling of melt technique, of $\mathrm{SeTePb}$ and $\mathrm{SeTeCd}$ have been chosen to study of dc volume conductivity in chalcogenide glasses. The chosen compositions $\left(\mathrm{Se}_{70} \mathrm{Te}_{30-\mathrm{x}} \mathrm{Cd}_{\mathrm{x}}(\mathrm{x}=1,2,3,4 \& 5)\right.$ and $\left.\mathrm{Se}_{85} \mathrm{Te}_{15-\mathrm{x}} \mathrm{Pb}_{\mathrm{x}}(\mathrm{x}=1,2,3 \& 4)\right)$ have been confirmed as amorphous using XRD. Bulk sample profile has been selected to study the volume dc conductivity and pellets of desired dimensions have been prepared under load of 5 tons. I-V characteristics of these pellets have been recorded using Keithley Electrometer / High resistance meter 6517A at different temperatures. This instrument was used in FVMI (Force Voltage Measure Current) mode, which provides the facility of use of single instrument to apply voltage and measure the current through sample. The decreasing nature of resistance with temperature confirms the semi conducting behavior of these glasses. The variation in conductivity with respect to concentration of the third element in ternary glass could be explained on the basis of formation of bonds of higher energy in the systems under test, which leads the system towards more theoretically dense structure. The observed linearity in $\operatorname{Ln}(\mathrm{I}) \mathrm{vs}^{1 / 2}$ curves, as per Jonschere and Hill relation, is suggestive of Poole-Frenkel type conduction in these glasses. The results are helpful in better conceptual understanding of physics for the conduction mechanism in such glasses and may be used for tailoring the end user application.
\end{abstract}

Keywords: chalcogenide glasses, semi-conducting glass, dc conductivity, poole- frenkel conduction
Volume 3 Issue I - 2019

\author{
Vibhav K Saraswat \\ Department of Physics, Indian Military Academy, Dehradun, India
}

Correspondence: Vibhav K Saraswat, Depaertment of Physics, ACC Wg, Indian Military Academy, Dehradun-248007 Uttarakhand, India, Tel +9l 9829485487,

Email vibhav.sps!@gmail.com

Received: August 04, 2018 | Published: February 01, 2019

\section{Introduction}

Selenium (Se) has proved its potential in wide commercial applications. Its device applications like switching, memory and Xeroxing etc. made it attractive. It also exhibits a unique property of reversible transformation. ${ }^{1}$ This property makes it very useful in optical memory devices. But in pure state it has some disadvantages because of its short lifetime and low sensitivity. To overcome these difficulties certain additives are used and binary and ternary chalcogenide glasses are obtained e.g. the addition of small percentage of $\mathrm{Sb}$ is sufficient to cause crystallization of $\mathrm{Se}^{2-4}$

Ternary chalcogenide glasses, with third additive as $\mathrm{Pb}$ and $\mathrm{Cd}$, have been interesting candidates among the researchers due to their potential applications in recent years. ${ }^{5-10}$ To the best of my knowledge, very few attempts have been made to study chalcogenide glasses with one of the component as $\mathrm{Pb}$ and $\mathrm{Cd}$. It may be due to the fact that lead is the last element in radioactive series, which is most stable, or lead is the one with which the formation of glass is most difficult deal. The addition of $\mathrm{Cd}$ also shows some interesting behavior, but it is hard to form glass with Cd because of its ductility. ${ }^{11}$ This motivated us to carry out dc electrical conductivity investigations on $\mathrm{Se}_{85} \mathrm{Te}_{15-\mathrm{x}} \mathrm{Pb}_{\mathrm{x}}(\mathrm{x}=$ $1,2,3 \& 4)$ and $\mathrm{Se}_{70} \mathrm{Te}_{30-\mathrm{x}} \mathrm{Cd}_{\mathrm{x}}(\mathrm{x}=1,2,3,4 \& 5)$ glasses.

\section{Experimental}

Glassy powders of $\mathrm{Se}_{70} \mathrm{Te}_{30-\mathrm{x}} \mathrm{Cd}_{\mathrm{x}}(\mathrm{x}=1,2,3,4 \& 5)$ and $\mathrm{Se}_{85} \mathrm{Te}_{15-\mathrm{x}} \mathrm{Pb}$ $(x=1,2,3 \& 4)$ have been obtained by grinding the ingots, prepared by melt quenching technique. ${ }^{12-15}$ Chalcogenide glasses belong to group of substances which have an incongruent melting point, exhibit a high vapour pressure during melting and are susceptible to oxidation and hydrolysis and therefore, the synthesis must be carried out in sealed evacuated quartz ampoule. The synthesis conditions are widely varied: they depend on the glass composition, glass forming ability (GFA) and highest temperature achieved during synthesis etc. The necessary chemical and physical purity ${ }^{12,16-18}$ of the prepared glass should be about $10^{-5} \mathrm{~mol} \%$ for the presence of $\mathrm{OH}, \mathrm{SH}$, SHe groups and the concentration of physical defects in the range $10^{2}-10^{3}$ per $\mathrm{cm}^{3}$. So, keeping all these in mind, the high purity constituent materials, purchased from Alfa Aesar, were weighed according to at.wt. \% and mixed. This mixture was sealed in a quartz ampoule, evacuated at $\sim 10^{-5}$ torr then this ampoule was heated up to $950^{\circ} \mathrm{C}$ for about 8 hours. During this period the ampoule was frequently shaken to achieve better homogeneity of melt. Finally, the ingot of the glassy material under test, after the ice-cooled water quenching of the ampoule, were obtained. So obtained ingots were grinded into fine powders.

The amorphous nature of these powders was confirmed by XRD $(\lambda=1.5406 \AA)$ machine, Bruker DX8 Advance. As a representative case XRD patterns of $\mathrm{SeTePb}$ glasses are shown in Figure 1. The pellets of $12 \mathrm{~mm}$ diameter and $1 \mathrm{~mm}$ thickness were prepared at 5 Ton load using a hydraulic machine, Metrex, New Delhi. These pellets were used to record I-V characteristics at different temperatures. I-V characteristics were recorded using Keithley Electrometer/ high resistance meter 6517A. The pellet was sandwiched between two copper electrodes with the help of pressure contact arrangement in an indigenously designed sample holder for the purpose. ${ }^{12}$ The same meter was used, in its FVMI mode, to apply the voltage across the sample and to measure the current. ${ }^{12}$ 


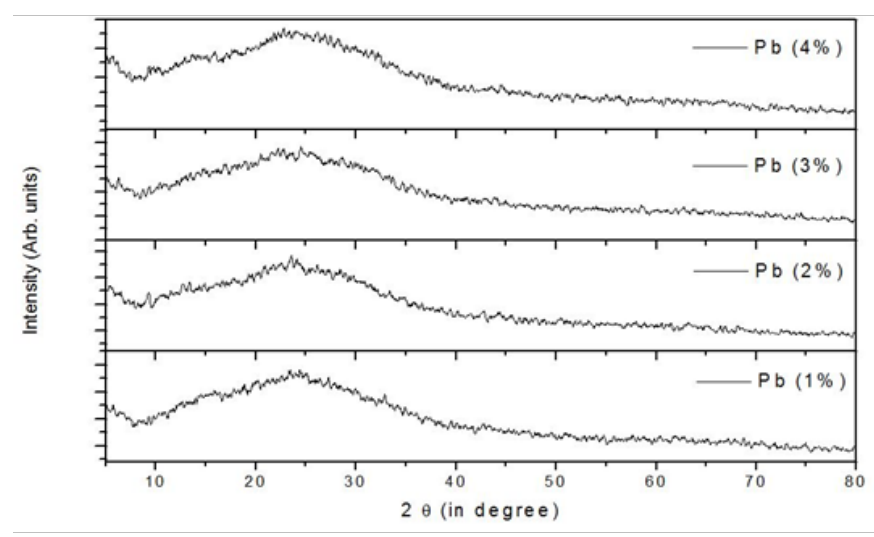

Figure I XRD patterns of $\mathrm{Se}_{85} \mathrm{Te}_{15-x} \mathrm{~Pb}_{x}(x=1,2,3$ \& 4) glasses.

\section{Results}

As a case of representation, Figures 2 \& 3 show the I-V characteristics of $\mathrm{Se}_{70} \mathrm{Te}_{25} \mathrm{Cd}_{5}$ and $\mathrm{Se}_{85} \mathrm{Te}_{11} \mathrm{~Pb}_{4}$ at different temperatures, respectively. It is quite evident from figures 2 and 3 that the resistance decreases with the increase in temperature, which is suggestive of the fact that these samples are of semiconducting nature. It was also observed that the composition $\mathrm{Se}_{70} \mathrm{Te}_{25} \mathrm{Cd}_{5}$ and $\mathrm{Se}_{85} \mathrm{Te}_{11} \mathrm{~Pb}_{4}$ show the maximum conduction at each temperature as compared to their counterparts in the respective series under test.

DC electrical conductivity has been calculated using a very basic relation $^{12}$

$$
\sigma_{D C}=(1 / R) \times(L / A)
$$

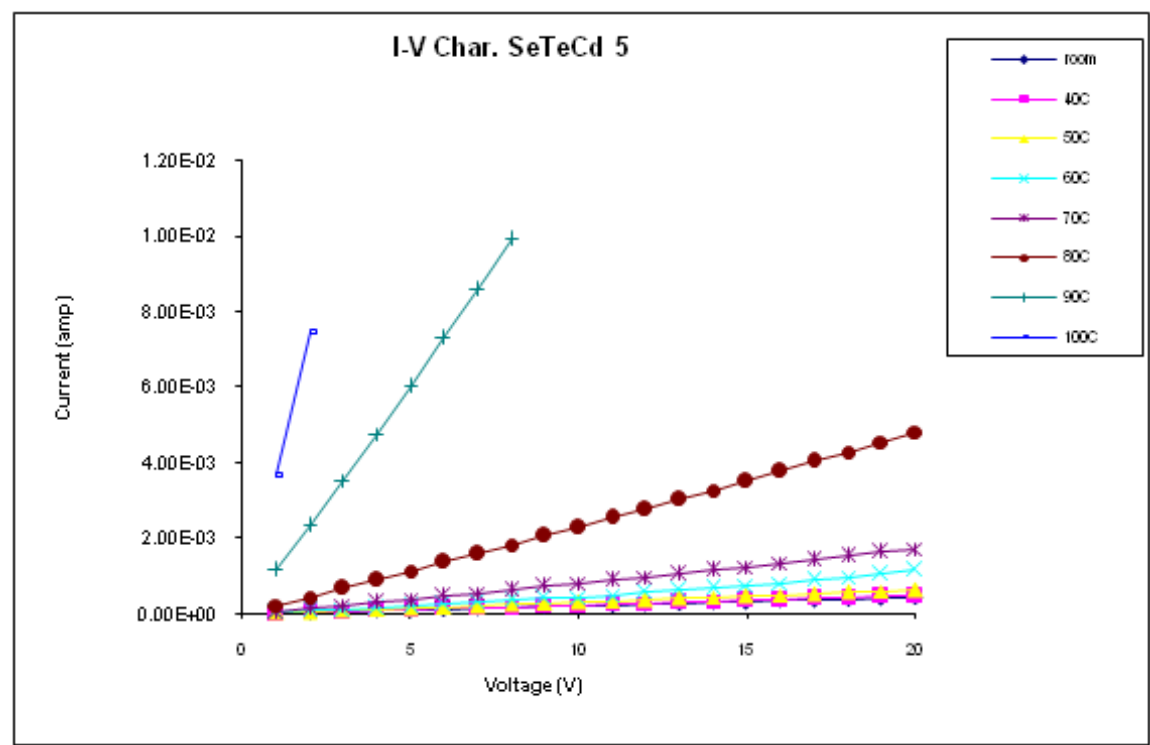

Figure $2 \mathrm{I}-\mathrm{V}$ Characteristics of $\mathrm{Se}_{70} \mathrm{Te}_{25} \mathrm{Cd}_{5}$ glass at different temperatures

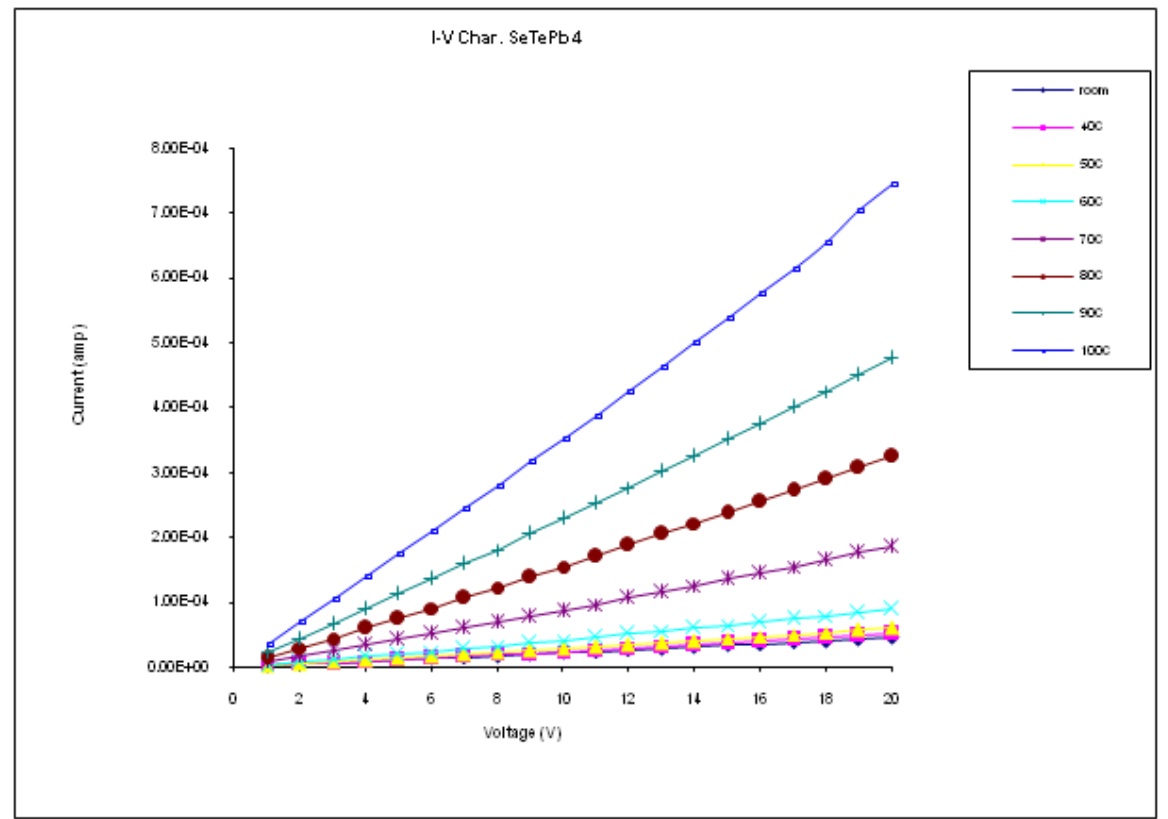

Figure $3 \mathrm{I}-\mathrm{V}$ Characteristics of $\mathrm{Se}_{85} \mathrm{Te}_{11} \mathrm{~Pb}_{4}$ glass at different temperatures. 
Figures 4 \& 5 show the variation in conductivity against the compositions of members of $\mathrm{SeTeCd}$ and $\mathrm{SeTePb}$ glassy series at different temperatures, respectively, as representative cases.

Figures $6 \& 7$ show the variation of $\mathrm{Ln}$ (I) with respect to $\mathrm{V}^{1 / 2}$, a consequence of relation between the current and the square root of the applied voltage as given by Jonschere ${ }^{19}$ for $\mathrm{Se}_{70} \mathrm{Te}_{25} \mathrm{Cd}_{5}$ and $\mathrm{Se}_{85} \mathrm{Te}_{11} \mathrm{~Pb}_{4}$ glasses, respectively and the same trend has been obtained for other members of the series.

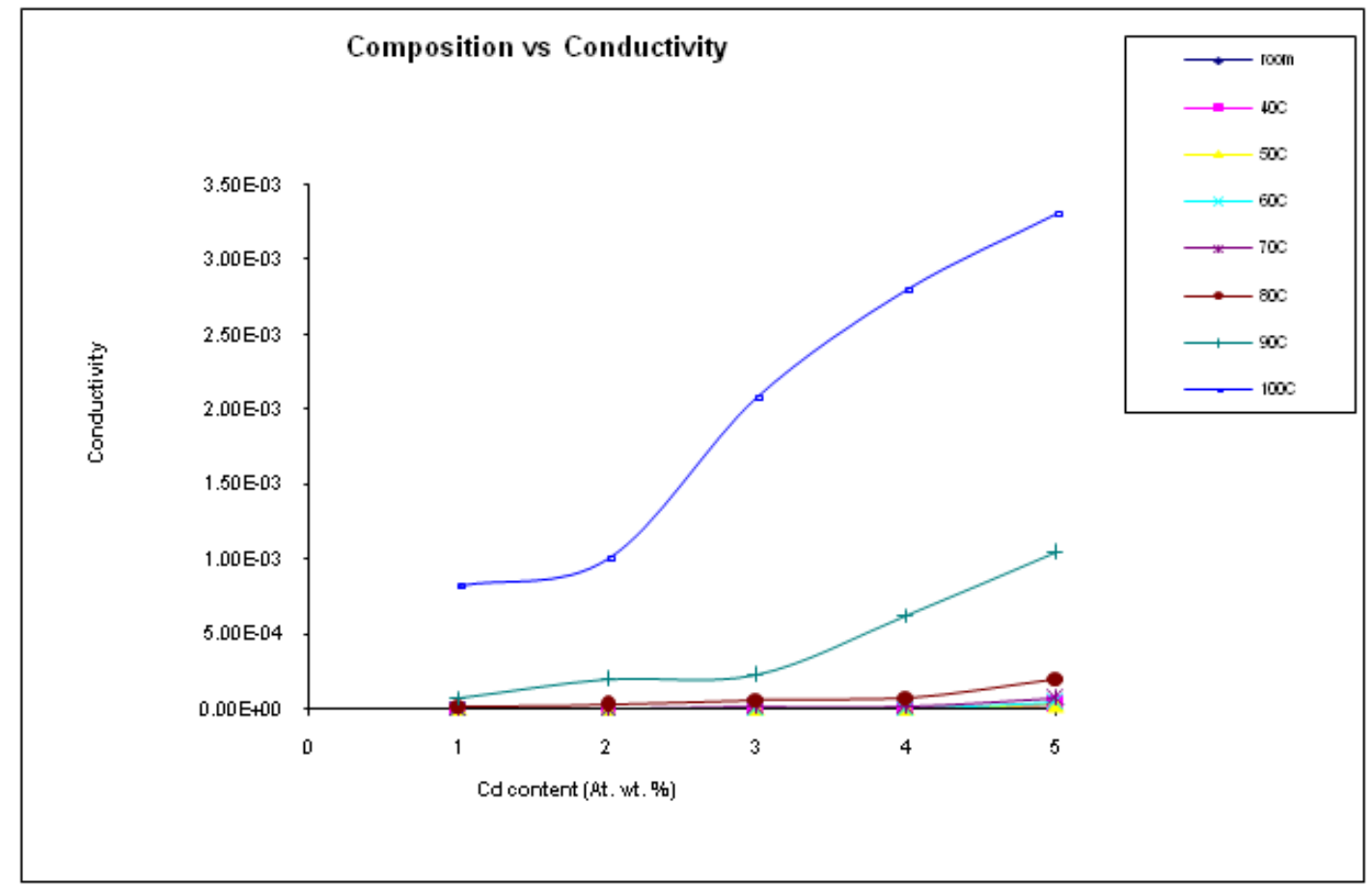

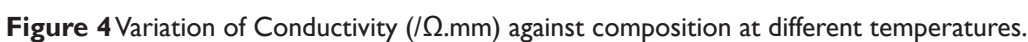

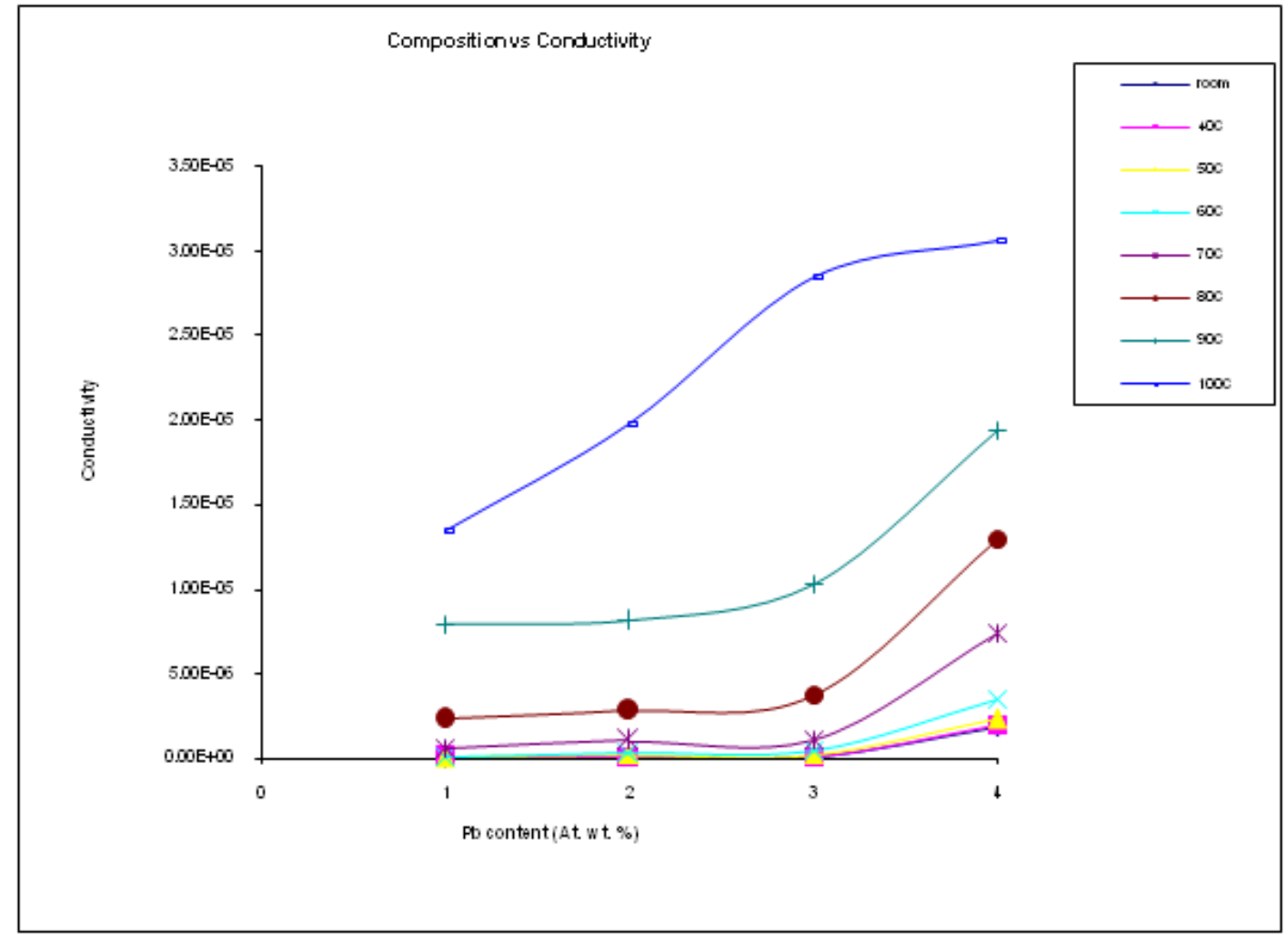

Figure 5 Variation of conductivity $(/ \Omega . \mathrm{mm})$ against composition at different temperatures. 


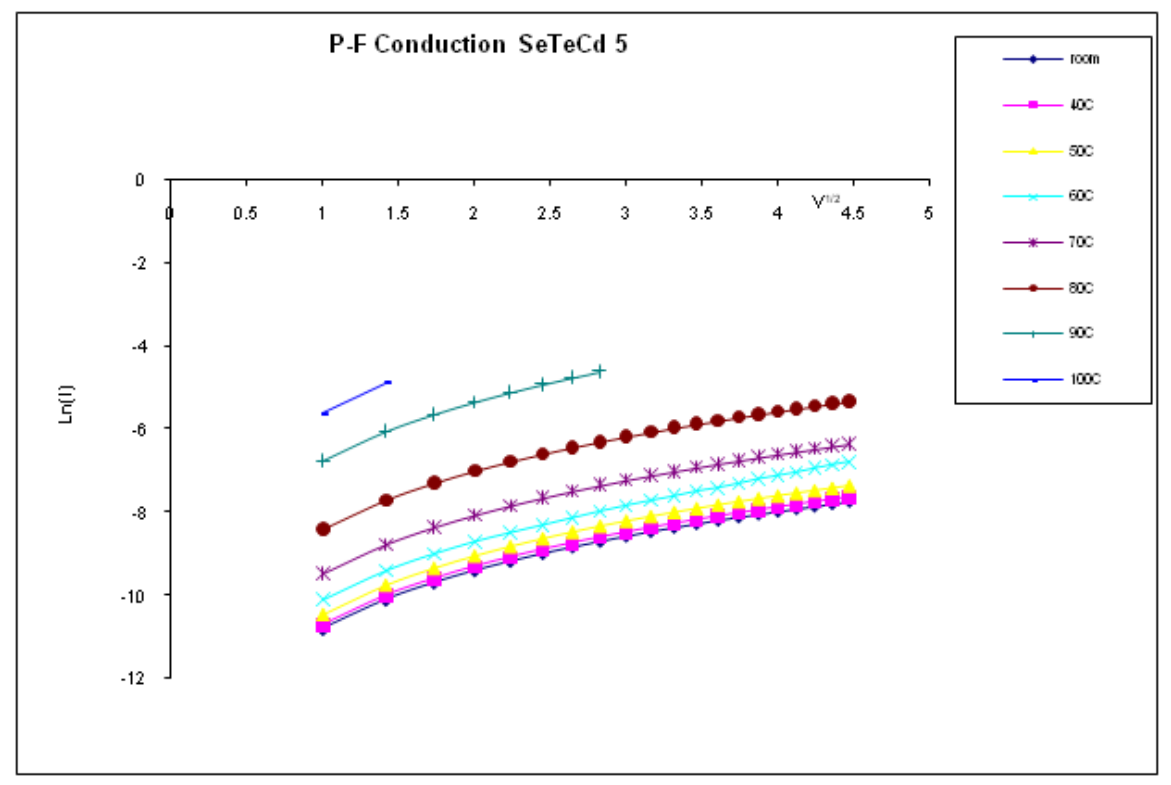

Figure 6 Verification of P-F conduction in $\mathrm{Se}_{70} \mathrm{Te}_{25} \mathrm{Cd}_{5}$ glass at different temperatures.

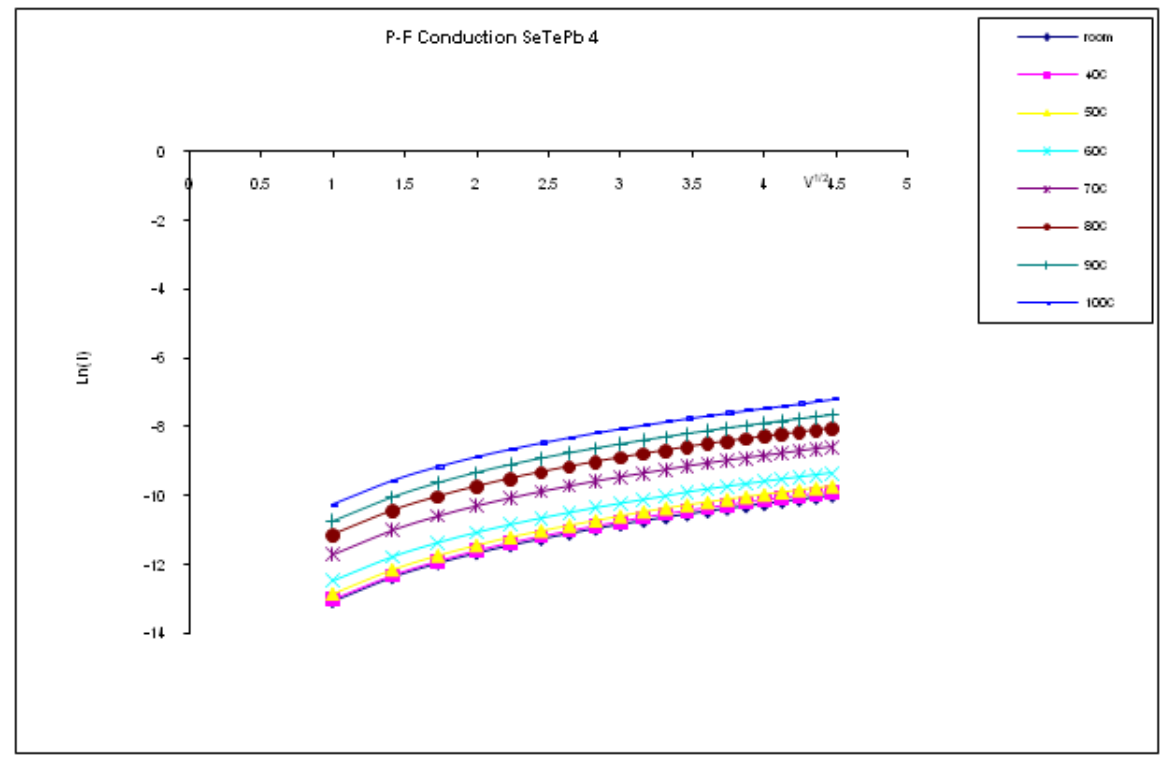

Figure 7 Verification of $\mathrm{P}-\mathrm{F}$ conduction in $\mathrm{Se}_{85} \mathrm{Te}_{11} \mathrm{~Pb}_{4}$ glass at different temperatures.

\section{Discussion}

There are two fundamental processes occurring in such semiconductors simultaneously namely, band conduction and hopping conduction. The band conduction occurs when the carriers are excited beyond the mobility edges into non-localized states at high temperatures. The excitation of the carriers into localized states at band edge causes the hopping conduction. ${ }^{12,20}$ Thus the total conductivity is given as,

$$
\sigma=\sigma_{i}+\sigma_{h}
$$

where, $\sigma_{i}$ is the intrinsic conductivity and $\sigma_{h}$ is the hopping conductivity.

The variation in conductivity can be explained on the basis of bond formation between the third element (i.e. $\mathrm{Cd}$ or $\mathrm{Pb}$, of the respective series) and Se \& Te. In glassy Se, about $40 \%$ of the atoms have ring structure and $60 \%$ of the atoms are bounded as polymeric chains. ${ }^{21}$ Tellurium enters as co-polymeric chains and tends to reduce the number of $\mathrm{Se}_{8}$ member rings. Simultaneously, it increases the number of $\mathrm{Se}$ and $\mathrm{Te}$ atoms in the chain structure. Now, if one adds the third element to such system, the third element atoms; $\mathrm{Cd}$ or $\mathrm{Pb}$ in present case, in SeTe system leads to the formation of bonds between Se \& $\mathrm{Cd} / \mathrm{Pb}$ and $\mathrm{Te} \& \mathrm{Cd} / \mathrm{Pb}$. In first series, $\mathrm{Se}_{70} \mathrm{Te}_{30-\mathrm{x}} \mathrm{Cd}_{\mathrm{x}}$ glasses, $\mathrm{Cd}$ is added at the cost of Te, which favours the formation of Cd-Se bonds in the system. The bond energy for Cd-Se bond is $127.6 \mathrm{~kJ} / \mathrm{mol}$ and for $\mathrm{Cd}-\mathrm{Te}$ bond is $100.0 \mathrm{~kJ} / \mathrm{mol}^{22}$ and hence the concentration of higher energy bonds of Cd-Se is increased as one adds $\mathrm{Cd}$ in the system.

For the second series, $\mathrm{Se}_{85} \mathrm{Te}_{15-\mathrm{x}} \mathrm{Pb}_{\mathrm{x}}$ glasses, the bond energy of $\mathrm{Pb}-\mathrm{Se}$ bond is $304.1 \mathrm{~kJ} / \mathrm{mol}$ and $\mathrm{Pb}-\mathrm{Te}$ bond is $252.0 \mathrm{~kJ} / \mathrm{mol}^{23}$. Since, $\mathrm{Pb}$ is added at the cost of Te which increases the probability for the 
formation of $\mathrm{Pb}$-Se bonds i.e concentration of $\mathrm{Pb}$-Se bonds increases with the increase of $\mathrm{Pb}$ content in $\mathrm{SeTePb}$ system resulting the more compact (theoretically dense) structure, also as in case of SeTeCd system. Hence, like first series, the conductivity increases with the increase of $\mathrm{Pb}$ content in the system.

Besides the formation of bond of higher energy, applied voltage, temperature and induced thermal effects due to higher voltage and other important factors contribute to the enhancement of electrical conduction in the samples.

Since chalcogenide glasses are semiconductors and the physical properties of these glasses strongly depend on various parameters, many researchers have floated ideas time to time to understand the conduction mechanism in such glasses. There are plenty of evidences that the conduction mechanism in chalcogenide glasses is of PooleFrenkel type. ${ }^{3,6,12,24-28}$ To confirm this, results of this study have been used.

The linearity of these curves, Figures $6 \& 7$ are suggestive of the fact that these glasses obey the Poole-Frenkel conduction mechanism. ${ }^{29}$ This linearity could be due to the absence of space charge resulting in a uniformity of field distribution between electrodes and practically, the current in such conduction remain unchanged if the polarities of the electrodes are reversed. This is due to the fact that current does not depend on the potential barrier at the interface ${ }^{30}$ The Poole-Frenkel conduction mechanism deals with the conduction in such materials where defect/impurity generated electrons \& traps are involved. The structural defects in the material cause additional energy states close to the band edge called 'traps'. These traps restrict the current flow because of a capture and emission process, thereby, becoming the dominant current mechanism. ${ }^{12,20}$

\section{Conclusion}

An important parameter, in electrical and sensing applications, DC electrical conductivity was calculated using I-V characteristics, recorded at different temperatures for both the glassy series i.e. $\mathrm{Se}_{70} \mathrm{Te}_{30-\mathrm{x}} \mathrm{Cd}_{\mathrm{x}}$ and $\mathrm{Se}_{85} \mathrm{Te}_{15-\mathrm{x}} \mathrm{Pb}_{\mathrm{x}}$ under test. It was found that the resistance decreases with the increasing temperature, which confirmed the semi-conducting nature of chosen samples. I-V characteristics and the conductivity variation with respect to the composition revealed that $\mathrm{Se}_{70} \mathrm{Te}_{25} \mathrm{Cd}_{5}$ and $\mathrm{Se}_{85} \mathrm{Te}_{11} \mathrm{~Pb}_{4}$ composition are most conducting compositions among the samples in their respective series at all temperatures. The variation in conductivity with composition could be explained on the basis of bond formation between the third element $(\mathrm{Cd} / \mathrm{Pb})$ and $\mathrm{Se} \& \mathrm{Te}$. The formation of higher energy bonds eventually leads to theoretically dense system, which reflects as the increase in conductivity. A comparative study of figures 2 and 3 also reveals that $\mathrm{SeTeCd}$ system is more conducting than that of $\mathrm{SeTePb}$ system. The linearity observed in Figures $6 \& 7$ confirm that these glasses possess Poole-Frenkel conduction mechanism.

\section{Acknowledgments}

Author is thankful to Prof. N.S. Saxena, Emrt. Scientist, University of Rajasthan, Jaipur, India for his valuable suggestions and facilities provided at SPS laboratory, University of Rajasthan, Jaipur, India.

\section{Conflicts of interest}

Author declares that there is no conflicts of interest related to work presented in this paper.

\section{References}

1. Tanaka K. Structural phase transitions in chalcogenide glasses. Phys Rev B. 1989;39:1270.

2. Moharram AH, Abu Sehly AA, Abu-El-Oyoun, et al. Pre-crystallization and crystallization kinetics of some Se-Te-Sb glasses Physica $B$ Condensed Matter. 2002;324(1-4):344-351.

3. Tanwar N, Saraswat YK, Saraswat Vibhav K. Enthalpy and entropy change during glass/crystal phasetransformation for $\mathrm{Ge}_{\mathrm{y}} \mathrm{Se}_{94-\mathrm{y}} \operatorname{In}_{6}(\mathrm{y}=10,15$ and20) glasses. Journal of Ovonic Research. 2015;11(4):183-188.

4. Tanwar Naveen, Saraswat Vibhav K. A study of kinetics of phase transformation of $\mathrm{Ge}_{10} \mathrm{Se}_{75} \mathrm{Sb}_{15}$ chalcogenide glass. $J$ of Non-crystalline Solids. 2014;394-395:1-5.

5. Wieder J, Aronson S. Properties of $\mathrm{Ge}_{10} \mathrm{Se}_{75} \mathrm{Te}$ glasses. $J$ of NonCrystalline Solids. 1979;33:405.

6. Saraswat Vibhav K, Tanwar Naveen, Saxena NS. Modifications in band gap \& dc conductivity of ternary chalcogenide glasses by addition of lead. Journal of Non-Oxide Glasses. 2012;4(4):55.

7. Deepika, Saraswat Vibhav K, Jain Praveen K, et al. Phase Transformation and Kinetics of Crystallization of $\mathrm{Ge}_{05} \mathrm{Sn}_{0.5} \mathrm{Se}_{25}$ Glass. AIP Conf Proceeding. 2008; 1004:85.

8. Clavaguera-Mora MT, Baro MD, Surinach S, et al. Enthalpy recovery in Se rich $\mathrm{Ge}_{10} \mathrm{Se}_{75}$ glasses during isothermal annealing and continuous heating. J of Non-Crystalline Solids. 1991;131:479.

9. Mahadevan S and Giridhar A. Coexistence of topological and chemical ordering effects in $\mathrm{Ge}_{\mathrm{x}} \mathrm{Ga}_{10} \mathrm{Se}_{90-\mathrm{x}}$ glasses. JNon Cryst Solids. 1993;152:42.

10. Maharjan NB, Saxena NS, Bhandari D. et al. Differential scanning calorimetry studies of $\mathrm{Se}_{85} \mathrm{Te}_{15-\mathrm{xb}} \mathrm{Pb}(\mathrm{x}=4,6,8$ and 10) glasses. Bull Mater Sci. 2000;23(5):369-375.

11. Saraswat Vibhav K, Kishore V, Saraswat YK, et al. Effect of Cd Addition in Band Gap and Volume Conductivity of SeTe Based Glasses. AIP Conference Proceeding. 2011;1391(1):504.

12. Saraswat Vibhav K, Singh K, Saxena NS, et al. Composition dependence of the electrical conductivity of $\mathrm{Se}_{85-\mathrm{x}} \mathrm{Te}_{15} \mathrm{Sb}_{\mathrm{x}}(\mathrm{x}=2,4,6,8$ and 10) glass at room temperature. Current Applied Physics. 2006;6:14.

13. Tanwar N, Saraswat YK, Saraswat Vibhav K. Calorimetric Study of Kinetics of Glass Transition and Crystallization of $\mathrm{Ge}_{7} \mathrm{Se}_{75} \mathrm{Sb}_{18}$ Chalcogenide Glass. Advanced Electrochemistry. 2014;2(1):71.

14. Tanwar Naveen, V Kishore, Ajay S Verma, et al. Study of Glass Transition Kinetics of $\mathrm{Ge}_{25-\mathrm{x}} \mathrm{Se}_{75} \mathrm{Sb}_{\mathrm{x}}(\mathrm{x}=12,15$ and 18) Glassy Alloys by Differential Scanning Calorimetery. Advanced Science Letters. 2016;22(11):3863-3866.

15. Tanwar Naveen, Saraswat Vibhav K. Study of Activation Energy of Crystallization and Growth Morphology of $\mathrm{Ge}_{25-\mathrm{x}} \mathrm{Se}_{75} \mathrm{Sb}_{\mathrm{x}}(\mathrm{x}=12$, 15, 18) Chalcogenide Glasses. Macromolecular Symposia. 2017;376(1):1600185.

16. Lezal D, Pedlikova J, Zavadil J, et al. Preparation and characterization of sulfide, selenide and telluride glasses. J of Non Crystalline Solids. 2003;326-327:47-52.

17. Tanwar Naveen, Saraswat Vibhav K. Kinetics of glass transition of $\mathrm{Ge}_{\mathrm{y}} \mathrm{Se}_{94-\mathrm{y}} \mathrm{In}_{6}(\mathrm{y}=10,15$ and 20$)$ chalcogenide glasses under nonisothermal condition. Ferroelectrics. 2017;519:131-138.

18. Saraswat Vibhav K, Tanwar Naveen. Investigation of Some Thermodynamic Parameters Reflecting Glass/Crystal PhaseTransformation of $\mathrm{Ge}_{25-\mathrm{x}} \mathrm{Se}_{75} \mathrm{Sb}_{\mathrm{x}}(\mathrm{x}=12,15,18)$ Glasses. Materials Focus. 2017;6:596-601.

19. Johschere AK, Hill RM, Hass GMH, et al. Physics of thin films. New York: Academic Press; 1975. p.152. 
20. Mott NF, Davis EA. Electronic processes in non-crystalline materials. 2nd ed. USA: Oxford University Press;1979. p. 590.

21. Reddy VK, Bhatnagar AK and Srivastava V. The influence of antimony impurity on optical and electrical properties of amorphous selenium. $J$ Phys D Condens Matt. 1992;4(23):5273.

22. Kerr JA, Stocker David W. CRC handbook of chemistry and physics. 81 ed. USA: CRC Press; 2001. p. 2556.

23. David RR. CRC handbook of chemistry and physics. 73rd ed. Ohio: Chemical rubber publishing; 1977. p. 346.

24. Saraswat Vibhav K, Kishore V, Singh K, et al. Electrical conductivity of Se-In chalcogenide glasses. Chalcogenide letters. 2006;3(8):61.

25. Dwivedi SK, Kumar A, Kumar S. High-field conduction in some chalcogenide glasses. Advance Materials for Optics and Electronics 1999;9:235-244.
26. Mehta N, Kumar A. Poole-Frenkel photoconductivity in amorphous $\mathrm{Se}_{75} \mathrm{Te}_{20} \mathrm{Sb}_{5}$ thin films. Glass Physics and Chemistry. 2010;36(3):313.

27. Abdel-Wahab F, Yelon A. Meyer-Neldel rule and Poole-Frenkel effect in chalcogenide glasses. J of Applied Physics. 2013;114:023707.

28. Kaes M, Salinga M. Impact of defect occupation on conduction in amorphous $\mathrm{Ge}_{2} \mathrm{Sb}_{2} \mathrm{Te}_{5}$. Scientific Report. 2016;6:31699.

29. Abdel Latif RM. DC Electrical measurements on evaporated thin films of vanadium pentoxide. Physica B. 1998;254(3-4):273-276.

30. Saraswat Vibhav K, Kishore V, Saxena NS, et al. I-V measurements of chalcogenide glass thin films. Ind JPure and ApplPhys. 2006;44:196-200. 